\title{
Risk Factors for Lymph Node Metastasis in Undifferentiated-Type Gastric Carcinoma
}

\author{
Myeong-Cherl Kook \\ Center for Gastric Cancer, National Cancer Center, Goyang, Korea
}

Undifferentiated-type carcinoma has a high incidence of lymph node metastasis. The independent risk factors for lymph node metastasis in undifferentiated-type carcinoma are invasion depth, tumor size, lymphovascular invasion, and presence of ulcer. In the cases that meet the curative resection criteria, no lymph node metastasis was observed in the Japanese studies, but some metastases were observed in Korean studies. After performing curative endoscopic submucosal dissection, the survival rate is similar to that of gastrectomy. The discrepancy between endoscopy and pathology is high in undifferentiated-type carcinoma. The tumor size in endoscopy is a significant risk factor for non-curative resection, and when the tumor size is small, the non-curative resection rate is significantly reduced. Lymphovascular invasion can be assessed in pathologic examination and D2-40 stain is helpful. The presence of ulcer should be determined by pathology, but ulcer's omission in pathology report makes the analysis difficult. Undifferentiatedtype carcinomas with differentiated-type components show higher lymph node metastasis rate than that of pure undifferentiatedtype carcinomas. The lymph node metastasis rate of signet ring cell type is lower than that of other undifferentiated-type carcinomas and is similar to differentiated-type carcinomas. The application of these additional histologic findings may improve the indication of endoscopic submucosal dissection. Clin Endosc 2019;52:15-20

Key Words: Undifferentiated-type carcinoma; Endoscopic mucosal resection; Lymph node metastasis

\section{INTRODUCTION}

Endoscopic submucosal dissection (ESD) has been introduced and performed as a treatment for a certain population with early gastric cancer (EGC) with a very low risk of lymph node metastasis (LNM). Gastric carcinoma can be grouped into differentiated-type carcinoma and undifferentiated-type carcinoma according to the differentiation degree. ${ }^{1}$ Undifferentiated-type histology is a risk factor of LNM in EGC. ${ }^{2}$ In the Japanese guideline, ESD for undifferentiated-type carcinoma is still an investigational treatment due to the high incidence

Received: October 26, 2018 Revised: December 31, 2018

Accepted: December 31, 2018

Correspondence: Myeong-Cherl Kook

Center for Gastric Cancer, National Cancer Center, 323 Ilsan-ro, Ilsandong-gu, Goyang 10408, Korea

Tel: +82-31-920-1745, Fax: +82-31-920-1369, E-mail: mckook@ncc.re.kr ORCID: https://orcid.org/0000-0002-3435-3301

cc This is an Open Access article distributed under the terms of the Creative Commons Attribution Non-Commercial License (http://creativecommons.org/ licenses/by-nc/3.0) which permits unrestricted non-commercial use, distribution, and reproduction in any medium, provided the original work is properly cited. of LNM. ${ }^{3}$ However, many researchers have tried to expand ESD indications, and there is an increasing interest in the suitability of ESD for undifferentiated-type carcinoma. This review will discuss the risk factors for LNM in undifferentiated-type carcinoma, the discrepancy of risk factors between pre- and post-ESD that should be considered when performing ESD, and the recently suggested pathologic factors that may be considered for more accurate indications.

\section{RISK FACTORS AND CURATIVE RESECTION CRITERIA}

Undifferentiated-type carcinoma has higher LNM rate than differentiated-type carcinoma. In mucosal carcinomas, the LNM rate is $4.2 \%-6.0 \%$ for undifferentiated-type carcinoma and $0.4 \%-1.8 \%$ for differentiated-type carcinoma, respectively. ${ }^{2,4-7}$ The independent risk factors for LNM in undifferentiated-type carcinoma are invasion depth, tumor size, and lymphovascular invasion (Table 1 ). ${ }^{8-11}$ When confined to 


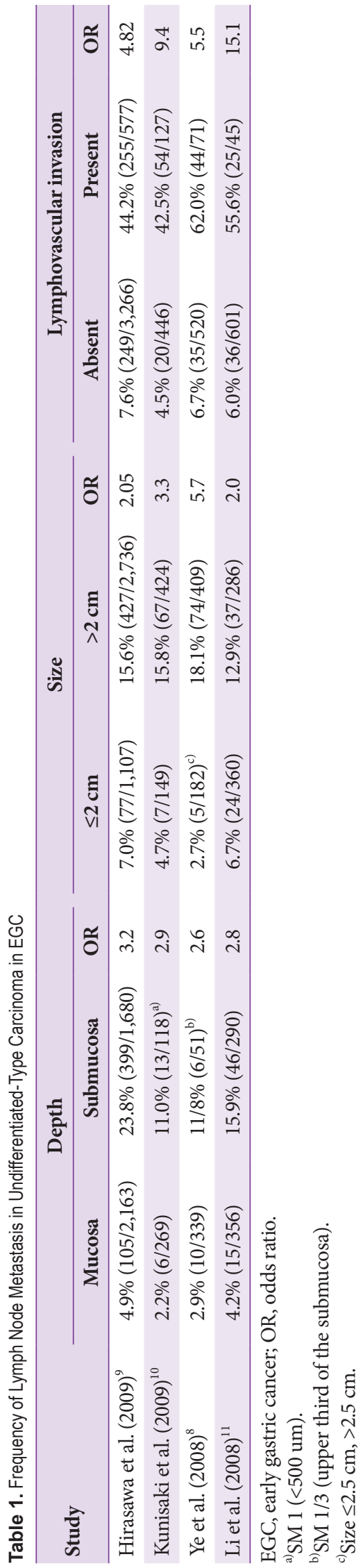

mucosal cancer, tumor size, lymphovascular invasion, and ulcer are the risk factors. ${ }^{9,11}$ Despite the high LNM rate in undifferentiated-type carcinoma as a whole, when it is confined in cases without any risk factor, the LNM rate can be decreased to a quite low level. According to the Japanese guideline, the curative resection criteria of ESD for undifferentiated-type carcinoma are the following: tumors that are confined to the mucosa, tumors that have no ulceration, tumor size $\leq 2 \mathrm{~cm}$, and absence of lymphovascular invasion. ${ }^{3}$ Within these criteria, no LNM was observed in the Japanese studies, but in some Korean studies, LNM was present, although at a low rate (Table 2). ${ }^{5,-111}$ These differences make the safety of performing ESD for undifferentiated-type carcinoma controversial.

Post-ESD survival is another important parameter in validating the effectiveness of ESD and can be used to address this controversy. In several retrospective studies from Korea and Japan, the occurrence of LNM, distant metastasis, or gastric cancer-related death was not observed when the post-ESD pathologic findings met the curative resection criteria. ${ }^{12-15}$ In the studies comparing ESD and gastrectomy using propensity score matching, there was no overall survival difference. ${ }^{16,17}$ These results suggest that ESD for undifferentiated-type carcinoma may be acceptable when curative resection is achieved. A prospective phase II clinical trial on the 5-year survival of ESD for undifferentiated-type carcinoma is currently under way in Japan. ${ }^{18}$

\section{DISCREPANCY OF THE RISK FACTORS BETWEEN PRE-ESD VS. POST-ESD FINDINGS}

The discrepancy of the risk factors between the pre-ESD endoscopy and the post-ESD pathology and the resulting

Table 2. Frequency of Lymph Node Metastasis in Undifferentiated-Type Carcinoma Meeting the Curative Resection Criteria ${ }^{a}$

\begin{tabular}{lcc}
\hline Study & Frequency & 95\% CI \\
\hline Gotoda et al. $(2000)^{5}$ & $0 \%(0 / 141)$ & $0 \%-2.6 \%$ \\
\hline Hirasawa et al. $(2009)^{9}$ & $0 \%(0 / 310)$ & $0 \%-0.96 \%$ \\
\hline Kunisaki et al. $(2009)^{10}$ & $0 \%(0 / 84)$ & - \\
Ye et al. $(2008)^{8}$ & $0 \%(0 / 119)^{\mathrm{b})}$ & - \\
\hline Li et al. $(2008)^{11}$ & $0.5 \%(1 / 201)^{\mathrm{c})}$ & - \\
${\text { Chung et al. }(2011)^{7}}^{8}$ & $1.1 \%(3 / 261)$ & $0 \%-2.4 \%$
\end{tabular}

CI, confidence interval.

${ }^{\text {a) }}$ Curative resection criteria: confined to the mucosa, lymphovascular invasion negative, ulcer negative, and size $\leq 2 \mathrm{~cm}$.

${ }^{\text {b) }}$ Size $\leq 2.5 \mathrm{~cm}$.

c)Ulcer was not evaluated. 
non-curative resection are considered important issues that are relevant for ESD. Undifferentiated-type carcinoma has higher discrepancy and lower curative resection rate than that of differentiated-type carcinoma. ${ }^{19}$ In a prospective study for differentiated-type carcinomas with absolute indication, other than size $(\leq 3 \mathrm{~cm})$, from Korea, the discrepancy rate was $20 \%$ overall, $8.7 \%$ for size, $6.9 \%$ for depth, $4.6 \%$ for ulcer, and $2.4 \%$ for histologic differentiation, ${ }^{20}$ and the curative resection rate was $86.1 \% .{ }^{21}$ In a prospective study for mucosal differentiated-type carcinoma with expanded indication from Japan, the curative resection rate was $67 \%{ }^{22}$ For undifferentiated-type carcinomas, such a prospective study result is not available yet. In retrospective studies, the discrepancy rates were $12.8 \%$ $16.8 \%$ for tumor size and $8.9 \%-22.4 \%$ for tumor depth, and the curative resection rate was $63.9 \%-70.3 \%$ when pre-ESD findings met the expanded indication (Table 3). 12,14,15

The tumor size in pre-ESD endoscopy is a significant risk factor for non-curative resection. The tumor size is not only a highly discrepant factor itself but also related to other risk factors, such as tumor depth, ulcer, and lymphovascular invasion, which could be assessed accurately after ESD. In a study for EGC with the absolute and expanded indications, the tumor size in pre-ESD endoscopy was smaller in the curative resection group than that in the non-curative resection group $(1.32 \mathrm{~cm}$ vs. $1.97 \mathrm{~cm}){ }^{19}$ For undifferentiated-type carcinoma, such a study using pre-ESD tumor size is not available, but there is a simulation study using gastrectomy cases comparing the endoscopic values and pathologic values. In this study, the cases that met the curative resection criteria had smaller endoscopic tumor size than the non-curative group $(1.08 \mathrm{~cm}$ vs. $1.30 \mathrm{~cm}$ ). ${ }^{23}$ For the studies using post-ESD pathologic size, the curative resection rates were $50 \%, 70 \%, 79 \%$, and $84 \%$ when the mean tumor sizes were $1.7 \mathrm{~cm}, 1.5 \mathrm{~cm}, 1.1 \mathrm{~cm}$, and 0.8 $\mathrm{cm}$, respectively. ${ }^{13,1,24,25}$ Therefore, if the tumor size criteria of ESD for undifferentiated-type carcinoma is set appropriately, non-curative resection rate may be reduced to a level similar to that of differentiated-type carcinoma.

Lymphovascular invasion is one of the important causes of non-curative resection, but it cannot be evaluated in pre-ESD examination. The incidence rate of lymphovascular invasion was $3.1 \%-4.8 \%$ in undifferentiated-type carcinomas, meeting the pre-ESD indication. ${ }^{12,14,15}$ However, these data would have been acquired based on hematoxylin-eosin (HE) stain, since immunohistochemical staining for lymphovascular invasion was not performed in daily practice. In a study of 78 gastric cancers, the detection of lymphovascular invasion was $27 \%$ by HE stain and $44 \%$ by immunohistochemistry. ${ }^{26}$ Even the lymphovascular invasion frequency is low in cancers with ESD indication, there may be a significant increase of lymphovascular invasion if immunohistochemistry is performed. If we use the data obtained by immunohistochemical staining for lymphovascular invasion, the parameters and cutoff values of ESD indication may be newly established. The most commonly used immunohistochemical stain is D2-40. D2-40 is a clone of the specific antibody for podoplanin, which is a transmembrane glycoprotein originally identified in kidney podocytes. ${ }^{27}$ It selectively stains the lymphatic endothelium and does not stain the vascular endothelium. ${ }^{28}$ Lymphatics are mainly distributed over the deep lamina propria, muscularis mucosae, and the upper part of the submucosa just beneath the muscularis mucosa (Fig. 1). ${ }^{26}$ In fact, the term "lymphovascular invasion" has been used traditionally because the lymphatics and the small-sized venules cannot be distinguished in HE-stained slides. Recently, lymphatic invasion and venous invasion are described separately in pathologic reports. However, still, the term "lymphatic invasion" means the involvement of a small and thin-walled vessel not distinguished in the lymphatics and venules, and the term "venous invasion" is only applied when the vein that has a large lumen and a muscular wall is involved. ${ }^{29}$ Actually, venous invasion is known to be not related to LNM. ${ }^{30}$ Therefore, D2-40 stain may be enough to examine the risk for LNM.

Ulcer is another factor contributing to the discrepancy between endoscopy and pathology. It can be evaluated on endoscopy, but the final diagnosis is based on histological findings showing the disruption of the muscularis mucosae and submucosal fibrosis including the healed state of ulcer. ${ }^{3}$ It is especially difficult to diagnose an ulcer in endoscopy in the case of scar caused by a previous ulcer (Fig. 2). ${ }^{31}$ Additionally, the presence of ulcer is not included as a mandatory content in a pathology report until now, and it is often omitted. For this reason, data from many studies often fail to accurately reflect the presence of ulcer, and the frequency varies greatly depending on the studies. When studying the curative resec-

Table 3. Curative Resection Rate and Frequency of the Non-Curative Factors in Undifferentiated-Type Carcinoma Meeting the Pre-ESD Indication

\begin{tabular}{lccccc}
\hline Study & Curative resection & Tumor size & Depth & \multicolumn{1}{c}{ LVI } & Ulcer \\
\hline Ahn et al. $(2016)^{15}$ & $70.3 \%(71 / 101)$ & $16.8 \%(17 / 101)$ & $8.9 \%(9 / 101)$ & $4.0 \%(4 / 101)$ & - \\
Oka et al. $(2014)^{14}$ & $64.8 \%(81 / 125)$ & $12.8 \%(16 / 125)$ & $22.4 \%(28 / 125)$ & $4.8 \%(6 / 125)$ & $8.8 \%(11 / 125)$ \\
\hline Abe et al. $(2013)^{12}$ & $63.9 \%(62 / 97)$ & $14.4 \%(14 / 97)$ & $19.6 \%(19 / 97)$ & $3.1 \%(3 / 97)$ & $9.3 \%(9 / 97)$ \\
\hline
\end{tabular}

ESD, endoscopic submucosal dissection; LVI, lymphovascular invasion. 
tion criteria of ESD, the authors will make sure that the presence of ulcer is based on the pathologic finding. If there is no description in pathology reports, the authors should get the information with pathology slide review or explain that the data are based on the endoscopic finding. Another problem is to distingush ulcer from biopsy-induced disruption and fibrosis. Usually, such a biopsy-induced change is sharply localized, and the disruption of the muscularis mucosae is not of full thickness. However, sometimes, it is very difficult to differen- tiate ulcer with biopsy-induced disruption and fibrosis, and in such cases, reviewing the differences between the initial and the ESD endoscopic findings is recommended.

\section{ADDITIONAL HISTOLOGIC FACTORS RELATED TO LYMPH NODE METASTASIS}

The frequency of LNM differs according to additional his-
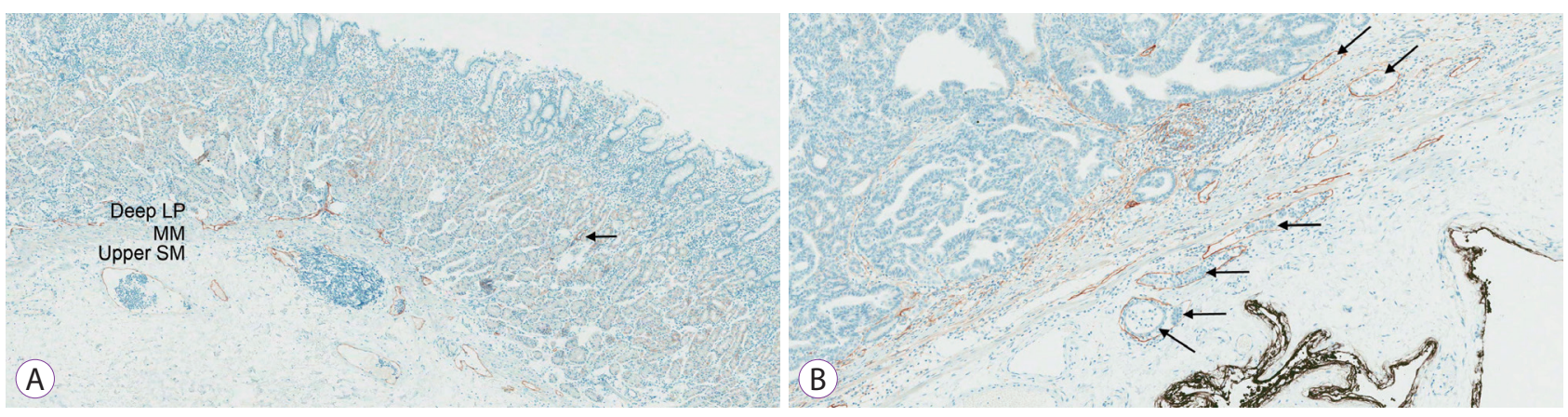

Fig. 1. Findings of D2-40 immunohistochemistry $(\times 100)$. (A) Distribution of lymphatics: The majority of lymphatic vessels are distributed over the deep lamina propria (deep LP), the muscularis mucosae (MM), and the upper layer of the submucosa (upper SM). A small number of lymphatic vessels can be present in the more superficial layer of the lamina propria (arrow). (B) Lymphatic invasion: The lymphatic vessels stained with D2-40 antibody are shown as brown-colored spaces and tumor cells (arrows) are present within the lumen.

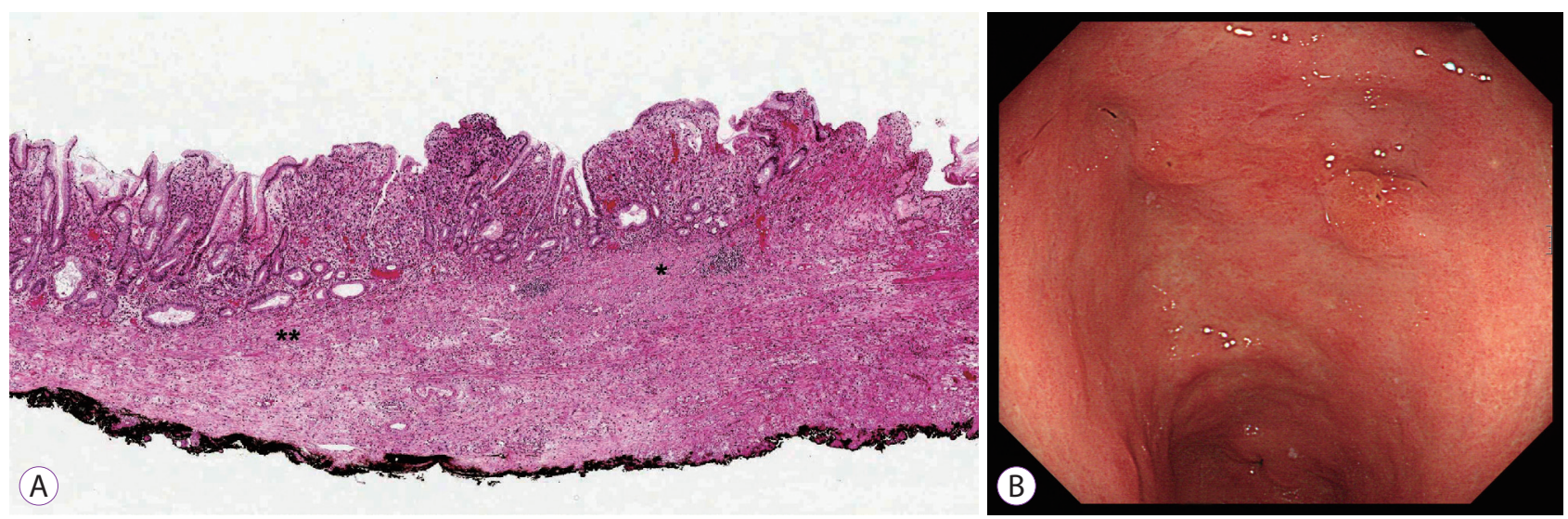

Fig. 2. Findings of ulcer. (A) Healed ulcer in a pathologic slide: Disruption and fibrous replacement $\left(^{*}\right)$ of the muscularis mucosae compared to the adjacent intact area ${ }^{* *}$ (hematoxylin-eosin stain, $\left.\times 40\right)$. (B) Endoscopic finding of (A): No definite ulcer change was diagnosed in endoscopy.

Table 4. Comparison of Lymph Node Metastasis Rates between Signet Ring Cell Type and Other Types in EGC

\begin{tabular}{lccc}
\hline Study & Differentiated-type & Signet ring cell type & Other undifferentiated-type $^{\text {a) }}$ \\
\hline Kwon et al. $(2014)^{34}$ & $11.7 \%(24 / 205)$ & $9.8 \%(5 / 51)$ & $17.1 \%(12 / 70)$ \\
Kim et al. $(2014)^{35}$ & $9.4 \%(99 / 1,024)$ & $9.0 \%(31 / 345)$ & $18.1 \%(118 / 651)$ \\
Ha et al. $(2008)^{36}$ & $8.2 \%(72 / 879)$ & $9.5 \%(37 / 388)$ & $24.9 \%(63 / 253)$ \\
Tong et al. $(2011)^{37}$ & $14.0 \%(32 / 229)$ & $14.7 \%(15 / 102)$ & $34.1 \%(31 / 91)$ \\
Guo et al. $(2015)^{38}$ & $9.8 \%(34 / 347)$ & $13.1 \%(26 / 198)$ & $23.8 \%(124 / 522)$ \\
\hline
\end{tabular}

EGC, early gastric cancer.

${ }^{\text {a) }}$ Poorly differentiated tubular adenocarcinoma or non-signet ring cell poorly cohesive carcinoma. 
tologic factors within the undifferentiated-type carcinoma. If the ESD is performed to the more selective subgroup rather than the entire undifferentiated-type carcinomas, there is a possibility of reducing the risk of LNM. These factors may be used to optimize ESD indications.

Recently, the difference between mixed histology and pure histology has received great attention. In routine practice, the histologic type is determined by the most dominant component. Therefore, the cases classified as undifferentiated-type carcinoma may contain minor differentiated-type components. At first glance, tumors composed of only pure undifferentiated-type components seem to be more malignant than those mixed with differentiated-type components, but actual results reveal the opposite. In a study for submucosal carcinomas, undifferentiated-type carcinomas with differentiated-type components showed higher LNM rate than that of pure undifferentiated-type carcinomas (36.5\% vs. $15.0 \%) .^{32}$ In a study for mucosal carcinomas, the same trend was observed $(19.0 \%$ vs. $6.0 \%){ }^{33}$ Given these results, the absence of minor differentiated-type component may be considered as an additional condition in optimizing the ESD indication of undifferentiated-type carcinoma.

Signet ring cell carcinoma and poorly differentiated tubular adenocarcinomas are both classified as undifferentiated-type carcinomas. However, the LNM rate of signet ring cell type is lower than that of the other undifferentiated-type carcinomas and even similar to differentiated-type carcinomas (Table 4). ${ }^{34-38}$ Additionally, compared to poorly differentiated tubular adenocarcinomas, signet ring cell carcinomas showed a lower incidence of submucosal invasion and deep margin involvement in ESD cases. ${ }^{13,39}$ These findings suggest that signet ring cell carcinoma can be more feasible for ESD than other undifferentiated-type carcinoma. One problem for signet ring cell carcinoma is that there is a discrepancy of classification criteria. Morphologically, the typical signet ring cell has an abundant intracellular mucin and eccentrical nucleus, but there are variant forms showing variable amounts of mucin and differing nuclear features. In the World Health Organization classification 2010 version, signet ring cell type was included in poorly cohesive carcinoma, and there has been more severe confusion about the entity of the signet ring cell type. To address these problems, an approach has been suggested to limit the diagnosis of signet ring cell carcinoma to the case with only typical signet ring cell morphology..$^{40}$ In a study comparing typical signet ring cell carcinomas and other poorly cohesive carcinomas, non-signet ring cell poorly cohesive carcinoma showed higher LNM rate, worse prognosis, and more frequent mutations than the typical signet ring cell carcinomas. ${ }^{41} \mathrm{~A}$ recent study for submucosal carcinomas reported that the cases composed of signet ring cell or poorly cohesive components mixed with minor tubular components have $31.9 \%$ of LNM rate, higher than $7.8 \%$ of the cases composed of purely signet ring cell or poorly cohesive components. ${ }^{42}$ These results suggest that a more strict application of the classification of signet ring cell carcinoma may improve the ESD indication, making it more accurate.

These factors and criteria described above are just presented, and verifying these factors and criteria regarding ESD indications is insufficient. Further research on the selected cases using these criteria is expected in the near future.

\section{CONCLUSIONS}

At present, the risk factors for LNM of undifferentiated-type carcinoma are invasion depth, tumor size, lymphovascular invasion, and ulcer. In cases where the current curative resection criteria have been met, a similar prognosis as gastric resection is observed, but a strict pathological examination is needed including the presence of lymphovascular invasion and ulcer. Some of the risk factors cannot be predicted accurately by pre-ESD endoscopy, and more limited indications may decrease the high frequency of non-curative resection. The use of histologic subtypes, such as mixed histology and pure typical signet ring cell histology, may be useful in the improvement of ESD indication.

\section{Conflicts of Interest}

The author has no financial conflicts of interest.

\section{REFERENCES}

1. Sugano H, Nakamura K, Kato Y. Pathological studies of human gastric cancer. Acta Pathol Jpn 1982;32 Suppl 2:329-347.

2. Choi KK, Bae JM, Kim SM, et al. The risk of lymph node metastases in 3951 surgically resected mucosal gastric cancers: implications for endoscopic resection. Gastrointest Endosc 2016;83:896-901.

3. Japanese Gastric Cancer Association. Japanese gastric cancer treatment guidelines 2010 (ver. 3). Gastric Cancer 2011;14:113-123.

4. Yamao $\mathrm{T}$, Shirao $\mathrm{K}$, Ono $\mathrm{H}$, et al. Risk factors for lymph node metastasis from intramucosal gastric carcinoma. Cancer 1996;77:602-606.

5. Gotoda T, Yanagisawa A, Sasako M, et al. Incidence of lymph node metastasis from early gastric cancer: estimation with a large number of cases at two large centers. Gastric Cancer 2000;3:219-225.

6. Seto Y, Shimoyama S, Kitayama J, et al. Lymph node metastasis and preoperative diagnosis of depth of invasion in early gastric cancer. Gastric Cancer 2001;4:34-38.

7. Chung JW, Jung HY, Choi KD, et al. Extended indication of endoscopic resection for mucosal early gastric cancer: analysis of a single center experience. J Gastroenterol Hepatol 2011;26:884-887.

8. Ye BD, Kim SG, Lee JY, et al. Predictive factors for lymph node metastasis and endoscopic treatment strategies for undifferentiated early gastric cancer. J Gastroenterol Hepatol 2008;23:46-50.

9. Hirasawa T, Gotoda T, Miyata S, et al. Incidence of lymph node metas- 
tasis and the feasibility of endoscopic resection for undifferentiated-type early gastric cancer. Gastric Cancer 2009;12:148-152.

10. Kunisaki C, Takahashi M, Nagahori Y, et al. Risk factors for lymph node metastasis in histologically poorly differentiated type early gastric cancer. Endoscopy 2009;41:498-503.

11. Li C, Kim S, Lai JF, et al. Risk factors for lymph node metastasis in undifferentiated early gastric cancer. Ann Surg Oncol 2008;15:764-769.

12. Abe S, Oda I, Suzuki H, et al. Short- and long-term outcomes of endoscopic submucosal dissection for undifferentiated early gastric cancer. Endoscopy 2013;45:703-707.

13. Kim JH, Kim YH, Jung DH, et al. Follow-up outcomes of endoscopic resection for early gastric cancer with undifferentiated histology. Surg Endosc 2014;28:2627-2633.

14. Oka S, Tanaka S, Higashiyama M, et al. Clinical validity of the expanded criteria for endoscopic resection of undifferentiated-type early gastric cancer based on long-term outcomes. Surg Endosc 2014;28:639-647.

15. Ahn JY, Park HJ, Park YS, et al. Endoscopic resection for undifferentiated-type early gastric cancer: immediate endoscopic outcomes and longterm survivals. Dig Dis Sci 2016;61:1158-1164.

16. Park JC, Lee YK, Kim SY, et al. Long-term outcomes of endoscopic submucosal dissection in comparison to surgery in undifferentiated-type intramucosal gastric cancer using propensity score analysis. Surg Endosc 2018;32:2046-2057.

17. Lee $\mathrm{S}$, Choi KD, Han M, et al. Long-term outcomes of endoscopic submucosal dissection versus surgery in early gastric cancer meeting expanded indication including undifferentiated-type tumors: a criteria-based analysis. Gastric Cancer 2018;21:490-499.

18. Takizawa K, Takashima A, Kimura A, et al. A phase II clinical trial of endoscopic submucosal dissection for early gastric cancer of undifferentiated type: Japan Clinical Oncology Group study JCOG1009/1010. Jpn J Clin Oncol 2013;43:87-91.

19. Kim EH, Park JC, Song IJ, et al. Prediction model for non-curative resection of endoscopic submucosal dissection in patients with early gastric cancer. Gastrointest Endosc 2017;85:976-983.

20. Kim JM, Sohn JH, Cho MY, et al. Pre- and post-ESD discrepancies in clinicopathologic criteria in early gastric cancer: the NECA-Korea ESD for early gastric cancer prospective study (N-Keep). Gastric Cancer 2016;19:1104-1113.

21. Kim SG, Park CM, Lee NR, et al. Long-term clinical outcomes of endoscopic submucosal dissection in patients with early gastric cancer: a prospective multicenter cohort study. Gut Liver 2018;12:402-410.

22. Hasuike N, Ono H, Boku N, et al. A non-randomized confirmatory trial of an expanded indication for endoscopic submucosal dissection for intestinal-type gastric cancer (cTla): the Japan Clinical Oncology Group study (JCOG0607). Gastric Cancer 2018;21:114-123.

23. Kwak DS, Min YW, Lee JH, et al. Outcomes of endoscopic submucosal dissection for early gastric cancer with undifferentiated-type histology: a clinical simulation using a non-selected surgical cohort. Gut Liver 2018;12:263-270.

24. Okada K, Fujisaki J, Yoshida T, et al. Long-term outcomes of endoscopic submucosal dissection for undifferentiated-type early gastric cancer. Endoscopy 2012;44:122-127.

25. Yamamoto Y, Fujisaki J, Hirasawa T, et al. Therapeutic outcomes of endoscopic submucosal dissection of undifferentiated-type intramucosal gastric cancer without ulceration and preoperatively diagnosed as 20 millimetres or less in diameter. Dig Endosc 2010;22:112-118.

26. Yonemura Y, Endou Y, Tabachi K, et al. Evaluation of lymphatic invasion in primary gastric cancer by a new monoclonal antibody, D2-40. Hum Pathol 2006;37:1193-1199.

27. Ordóñez NG. Podoplanin: a novel diagnostic immunohistochemical marker. Adv Anat Pathol 2006;13:83-88.

28. Pusztaszeri MP, Seelentag W, Bosman FT. Immunohistochemical expression of endothelial markers CD31, CD34, von Willebrand factor, and Fli-1 in normal human tissues. J Histochem Cytochem 2006;54:385395.

29. Kim WH, Park CK, Kim YB, et al. A standardized pathology report for gastric cancer. Korean J Pathol 2005;39:106-113.

30. Fujimoto A, Ishikawa Y, Akishima-Fukasawa Y, et al. Significance of lymphatic invasion on regional lymph node metastasis in early gastric cancer using LYVE-1 immunohistochemical analysis. Am J Clin Pathol 2007;127:82-88

31. Park SM, Kim BW, Kim JS, Kim YW, Kim GJ, Ryu SJ. Can endoscopic ulcerations in early gastric cancer be clearly defined before endoscopic resection? A survey among endoscopists. Clin Endosc 2017;50:473-478.

32. Hanaoka N, Tanabe S, Mikami T, Okayasu I, Saigenji K. Mixed-histologic-type submucosal invasive gastric cancer as a risk factor for lymph node metastasis: feasibility of endoscopic submucosal dissection. Endoscopy 2009;41:427-432.

33. Takizawa K, Ono H, Kakushima N, et al. Risk of lymph node metastases from intramucosal gastric cancer in relation to histological types: how to manage the mixed histological type for endoscopic submucosal dissection. Gastric Cancer 2013;16:531-536.

34. Kwon KJ, Shim KN, Song EM, et al. Clinicopathological characteristics and prognosis of signet ring cell carcinoma of the stomach. Gastric Cancer 2014;17:43-53.

35. Kim BS, Oh ST, Yook JH, Kim BS. Signet ring cell type and other histologic types: differing clinical course and prognosis in T1 gastric cancer. Surgery 2014;155:1030-1035.

36. Ha TK, An JY, Youn HK, Noh JH, Sohn TS, Kim S. Indication for endoscopic mucosal resection in early signet ring cell gastric cancer. Ann Surg Oncol 2008;15:508-513.

37. Tong JH, Sun Z, Wang ZN, et al. Early gastric cancer with signet-ring cell histologic type: risk factors of lymph node metastasis and indications of endoscopic surgery. Surgery 2011;149:356-363.

38. Guo CG, Zhao DB, Liu Q, et al. Risk factors for lymph node metastasis in early gastric cancer with signet ring cell carcinoma. J Gastrointest Surg 2015;19:1958-1965.

39. Bang CS, Park JM, Baik GH, et al. Therapeutic outcomes of endoscopic resection of early gastric cancer with undifferentiated-type histology: a Korean ESD registry database analysis. Clin Endosc 2017;50:569-577.

40. Mariette C, Carneiro F, Grabsch HI, van der Post RS, Allum W, de Manzoni G. Consensus on the pathological definition and classification of poorly cohesive gastric carcinoma. Gastric Cancer 2019;22:1-9.

41. Kwon CH, Kim YK, Lee S, et al. Gastric poorly cohesive carcinoma: a correlative study of mutational signatures and prognostic significance based on histopathological subtypes. Histopathology 2018;72:556-568.

42. Kim YH, Park JH, Park CK, et al. Histologic purity of signet ring cell carcinoma is a favorable risk factor for lymph node metastasis in poorly cohesive, submucosa-invasive early gastric carcinoma. Gastric Cancer 2017;20:583-590. 